Chirurg 2013 $\cdot 84: 61$

DOI 10.1007/s00104-012-2437-0

Online publiziert: 16. Dezember 2012

c) Springer-Verlag Berlin Heidelberg 2012

O. Strobel · M.W. Büchler

Klinik für Allgemein-, Viszeral- und Transplantationschirurgie, Universität Heidelberg

\title{
"Liver-first" bei synchron hepatisch metastasiertem kolorektalem Karzinom?
}

4. Oxaliplatin- und/oder Irinotecan-basierte Chemotherapie vor der Leberresektion,

Andres A, Toso C, Adam R et al (2012) A survival analysis of the liver-first reversed management of advanced simultaneous colorectal liver metastases: a LiverMetSurvey-based study. Ann Surg 256:772-779

\section{Hintergrund und Fragestellung}

Etwa 25\% der Patienten mit kolorektalem Karzinom haben synchrone Lebermetastasen. Bei Patienten mit nichtstenosierendem Tumor und Lebermetastasen ist unklar, in welcher Reihenfolge die Therapie erfolgen sollte. Beim klassischen Management (KM) erfolgt die Resektion des Primärtumors gefolgt von Chemotherapie und einer Leberresektion im Verlauf, falls diese technisch möglich ist (kein Progress, Downstaging primär irresektabler Metastasen). Insbesondere für Patienten mit multiplen Lebermetastasen wurde als mögliche Alternative ein umgekehrtes Vorgehen („reversed management“, RM) beschrieben mit primärer Chemotherapie, gefolgt von einer Resektion der Lebermetastasen und zuletzt der Resektion des Primärtumors.

Andres et al. verglichen diese beiden Ansätze basierend auf einem prospektiven internationalen Register.

\section{Methoden}

Einschlusskriterien waren:

1. kolorektales Karzinom mit mindestens 2 synchronen Lebermetastasen,

2. zweizeitiges Vorgehen mit einem Abstand zwischen kolorektaler und Leberresektion von mehr als 10 Tagen,

3. Eingriffe zwischen 2000 und 2010,
5. erfolgreiche R0-Resektion des Primärtumors und der Lebermetastasen.

Zielparameter waren das Gesamt- und das rezidivfreie Überleben.

\section{Ergebnisse}

Es wurden 787 Patienten eingeschlossen: 729 in die KM-Gruppe und 58 in die RM-Gruppe. Die Gruppen waren bezüglich epidemiologischer Daten, Größe des Primärtumors und Ausmaß der Lebermetastasierung (Größe, Anzahl, Lokalisation, Fong-Score) vergleichbar. In der RM-Gruppe war der Anteil der Patienten mit Rektumkarzinom, mit neoadjuvanter Radiotherapie vor Kolektomie und mit einer Oxaliplatin- und Irinotecan-haltigen Chemotherapie signifikant höher, die Rate von Lymphknotenmetastasen signifikant niedriger (letzteres vermutlich durch Downstaging). Das Gesamtüberleben und das rezidivfreie Überleben nach 5 Jahren waren in der KM-Gruppe und der RM-Gruppe vergleichbar ( $46 \%$ vs. $48 \%$, $26 \%$ vs. $30 \%)$.

\section{Diskussion}

Die Autoren folgern, dass KM und RM bei synchronen kolorektalen Lebermetastasen zu vergleichbaren Langzeitergebnissen führen, sofern die jeweilige Strategie erfolgreich abgeschlossen werden kann (R0-Resektion aller Manifestationen).

Der Nutzen eines KM oder RM hängt allerdings ganz wesentlich davon ab,

- bei welchem Anteil der Patienten die jeweilige Strategie erfolgreich abgeschlossen werden kann,
- wie das Überleben der Patienten ist, bei denen keine R0-Resektion erreicht werden kann und

- zu welchen Ergebnissen dies für die Gesamtheit der Patienten führt, bei denen eine KM oder RM begonnen wurde.

Diese grundlegenden Fragen bleiben aufgrund des Designs der vorliegenden Studie völlig offen und müssen nun in randomisiert-kontrollierten Studien geklärt werden.

Das Design solcher Studien sollte Subgruppen berücksichtigen, die mehr von der einen oder anderen Strategie profitieren könnten. Wie die Verteilung der Primärtumoren in der vorliegenden Studie suggeriert, könnten Patienten mit Rektumkarzinom von einem RM profitieren, weil bei KM eine neoadjuvante Therapie vor Rektumresektion zur einer zusätzlichen Verzögerung der Leberresektion führt. Zudem sollte die Option einer synchronen Kolon- und Leberresektion einbezogen werden und zwar sowohl bei limitierter Lebermetastasierung als auch bei multiplen Metastasen, die im Rahmen eines mehrzeitigen Vorgehens resektabel scheinen.

\section{Korrespondenzadresse}

\section{PD Dr. O. Strobel}

Klinik für Allgemein-, Viszeral- und Transplantationschirurgie, Universität Heidelberg,

Im Neuenheimer Feld 110, 69120 Heidelberg Oliver.Strobel@med.uni-heidelberg.de

Interessenkonflikt. Der korrespondierende Autor gibt für sich und seinen Koautor an, dass kein Interessenkonflikt besteht. 